07,14

\title{
Особенности статистических распределений прочностей моно- и полифиламентных ультраориентированных высокопрочных волокон сверхвысокомолекулярного полиэтилена
}

\author{
(C) Ю.М. Бойко ${ }^{1}$, В.А. Марихин ${ }^{1}$, О.А. Москалюк ${ }^{2}$, Л.П. Мясникова ${ }^{1}$ \\ ${ }^{1}$ Физико-технический институт им. А.Ф. Иоффре РАН, \\ Санкт-Петербург, Россия \\ ${ }^{2}$ Санкт-Петербургский государственный университет технологии и дизайна, \\ Санкт-Петербург, Россия \\ E-mail: yuri.boiko@mail.ioffe.ru
}

Поступила в Редакцию 25 ноября 2019 г.

В окончательной редакции 25 ноября 2019 г.

Принята к публикации 25 ноября 2019 г.

\begin{abstract}
Закономерности статистических распределений прочности при разрыве высокопрочных сверхориентированных мононитей и полифиламентных волокон, состоящих из нескольких сотен единичных волокон, сверхвысокомолекулярного полиэтилена (СВМПЭ) проанализированы с помощью моделей Вейбулла и Гаусса на базе большого массива измерений (по 50 идентичных образцов в каждой серии). Показано, что распределение прочности полифиламентных волокон СВМПЭ может быть описано как в рамках нормального распределения Гаусса, так и при использовании стандартной функции распределения Вейбулла. В то же время, распределение прочности мононитей СВМПЭ, вытянутых до предельной степени вытяжки 120, не подчиняется нормальному распределению. Обсуждены причины выявленного различия типов статистических распределений прочностей моно- и полифиламентов высокопрочного СВМПЭ при разрушении.
\end{abstract}

Ключевые слова: сверхвысокомолекулярный полиэтилен, гель-формование, ориентационная вытяжка, прочность, статистика.

DOI: 10.21883/FTT.2020.04.49125.637

\section{1. Введение}

Высокоориентированные волокна и нити из сверхвысокомолекулярного полиэтилена (СВМПЭ), полученные по гель-технологии, являются перспективными материалами, так как они впервые продемонстрировали возможность достижения очень высоких значений прочности $(\sigma)$ и модуля упругости $(E)$ при растяжении для гибкоцепных полимеров [1-6], вплоть до $\sigma=6 \mathrm{GPa}$ и $E=225 \mathrm{GPa}$ [7] для образцов, полученных в лабораторных условиях. Эти значения $\sigma$ и $E$ близки к показателям современных неорганических материалов углеродных [8], стеклянных и кварцевых волокон [9], являющихся основными конструкционными материалами, находящими широкое практическое применение в современных областях, требующих особую надежность при эксплуатации - бронезащите живой силы и техники, ракетостроении, авиации и т.д. При этом важно отметить, что по удельным характеристикам (на единицу массы материала) гель-волокна СВМПЭ являются „рекордсменами“ среди всех типов сверхпрочных материалов.

Однако, достигнутые на сегодняшний день физикомеханические характеристики гель-волокон из СВМПЭ как для лабораторных образцов, так и для промышленных волокон СВМПЭ типа Dyneema и Spectra ведущих фирм DSM (Нидерланды) и Honeywell (США), существенно ниже теоретических оценок $[1,2]$. Для вы- яснения физических причин этих расхождений и поиска научно-обоснованных путей дальнейшего совершенствования метода гель-технологии представляется важным выяснить закономерности статистического распределения механических характеристик.

При анализе больших массивов данных довольно часто используют нормальное распределение или распределение Гаусса (см., например, [10]), предполагающее равновероятностный характер разрушения в объеме образца. С другой стороны, для хрупких материалов, разрушение которых связывают с наличием дефектов поверхностных трещин, наиболее перспективной оказалась модель Вейбулла [8,9,11-28].

Сверхпрочные гель-волокна и нити СВМПЭ характеризуются наиболее высокими в мировой практике значениями прочности для гибкоцепных полимеров, и их по всем признакам можно также отнести к хрупким материалам, несмотря на их полимерную „принадлежность“. В этой связи в наших предыдущих работах [29-31] впервые было показано, что на примере особо прочных и сверхвысокомодульных мононитей СВМПЭ, полученных в лабораторных условиях, действительно возможно применение статистики Вейбулла для надежного описания распределения не только прочности, но и двух других не менее важных механических характеристик модуля упругости $E$ и деформации при разрыве $\left(\varepsilon_{\mathrm{b}}\right)$. Кроме того, удалось также выявить причину возник- 
новения поверхностных трещин - образование особых ротационных дефектов - полос сброса, провоцирующих развитие трещин на поверхности мононитей [29].

Вместе с тем, необходимо отметить, что для анализа поведения хрупких и квазихрупких материалов обычно не используют статистику Гаусса. В то же время, представляется важным сопоставить два типа распределений - Гаусса и Вейбулла - для анализа механического поведения образцов специфического типа, существенных для практического применения, в виде пучка, состоящего из большого количества (обычно, из нескольких сотен) индивидуальных моноволокон малого поперечного сечения (единицы $\mu m$ ). Для этих образцов разрушение одного из таких моноволокон не обязательно должно приводить к разрушению всего образца, так как прочность образца в таком случае уменьшится всего лишь менее чем на $1 \%$. Поэтому нельзя исключить возможность корректного описания распределения прочности таких образцов и при использовании зависимости Гаусса. Действительно, недавно нами [32] было показано, что распределение прочности при растяжении полифиламентных волокон полиамида-6, состоящих из двухсот отдельных волокон, может быть описано не только при использовании стандартной функции распределения Вейбулла, но и в рамках модели Гаусса. Иными словами, проявление этого эффекта продемонстрировало дуализм статистического распределения прочности полифиламентного полимерного материала.

В этой связи возникает важный вопрос: возможно ли проявление подобного дуализма при описании статистики распределения прочности особо прочных полимерных материалов? Действительно, ни для моноволокон, ни для полифиламентов сверхпрочных полимеров этот вопрос не исследовался. Следовательно, выяснение существования дуализма статистического распределения прочности особо прочных полимерных моно- и полифиламентов, заключающегося в возможности одновременного корректного описания распределения $\sigma$ как в рамках модели Вейбулла, так и в рамках модели Гаусca, или отсутствия такового представляется актуальной задачей. Для ее решения в настоящей работе при использовании функций Вейбулла и Гаусса были исследованы распределения большого массива данных по прочностям высокопрочных пленочных мононитей и полифиламентных волокон, сформованных из гелей СВМПЭ.

\section{2. Статистика Вейбулла}

В статистике Вейбулла кумулятивная функция вероятности $P(\sigma)$, описывающая вероятность разрушения идентичных образцов при данном или более низком напряжении $\sigma$, определяется как

$$
P(\sigma)=1-\exp \left[-\left(\sigma / \sigma_{0}\right)^{m}\right],
$$

где $m$ - так называемый модуль Вейбулла, который является лишь мерой дисперсии данных, $\sigma_{0}-$ масштаб- ный параметр (в идеальном случае, он должен совпадать со средним значением прочности [26]). Для проведения анализа в рамках модели Вейбулла, массив результатов испытаний упорядочивается в порядке возрастания величины механической характеристики и преобразуется затем в экспериментальное распределение вероятностей. В этом случае $j$-у результату в массиве данных из $n$ образцов присваивается совокупная вероятность разрушения $\left(P_{\mathrm{j}}\right)$. Для расчета этих вероятностей используются довольно простые соотношения $P_{\mathrm{j}}=f(j, n)$ - так называемые определители. Одним из наиболее корректных определителей является уравнение (2), предложенное в [14]:

$$
P_{\mathrm{j}}=(j-0.5) / n \text {. }
$$

После двойного логарифмирования левой и правой частей уравнения $(1)$ и замены $P(\sigma)$ на $P_{\mathrm{j}}$ получаем уравнение (3)

$$
\ln \ln \left[1 /\left(1-P_{\mathrm{j}}\right)\right]=-m \cdot \ln \sigma_{0}+m \cdot \ln \sigma .
$$

Уравнение (3) представляет собой достаточно простое линейное уравнение

$$
y=a+b x,
$$

где $\quad y=\ln \ln \left[1 /\left(1-P_{\mathrm{j}}\right)\right], \quad a=-m \cdot \ln \sigma_{0}, \quad b=m \quad$ и $x=\ln \sigma$. Определив $m$ как угол наклона зависимости $\ln \ln \left[1 /\left(1-P_{\mathrm{j}}\right)\right]=f(\ln \sigma) \quad$ с $\quad$ помощью $\quad$ стандартной процедуры линейного регрессионного анализа, можно определить параметр $\sigma_{0}$ путем решения уравнений (5) и (6)

$$
\begin{gathered}
\ln \sigma_{0}=-a / m, \\
\sigma_{0}=\exp (-a / m) .
\end{gathered}
$$

\section{3. Экспериментальная часть}

\section{1. Образцы}

Исследовались три серии образцов. Первая и вторая серии образцов представляли собой ультраориентированные высокопрочные пленочные мононити СВМПЭ (средневязкостная молекулярная масса $3000 \mathrm{~kg} / \mathrm{mol}$ ) со степенью вытяжки $\lambda=120$, полученные на лабораторной установке методом многостадийной зонной вытяжки [1] ксерогелей СВМПЭ, сформованных из растворов в декалине и минеральном масле [29]. Третья серия образцов представляла собой полифиламентные волокна Dyneema SK60, состоящие из нескольких сотен единичных волокон, любезно предоставленные фирмой DSM, Нидерланды.

\section{2. Механические испытания}

Для определения прочности при разрыве $\sigma$ образцы подвергались растяжению на разрывной машине Instron-1122 при комнатной температуре при скорости растяжения $10 \mathrm{~mm} / \mathrm{min}$ и длине рабочей части $10 \mathrm{~mm}$ 
для одиночных нитей, и при скорости растяжения $200 \mathrm{~mm} / \mathrm{min}$ и длине рабочей части $50 \mathrm{~cm}$ для полифиламентов. Для получения статистически достоверных результатов были протестированы по 50 идентичных образцов пленочных мононитей (для каждого из двух использованных растворителей) [29] и полифиламентов, т. е. всего 150 образцов.

\section{4. Результаты и их обсуждение}

Для выяснения возможности описания в рамках модели Гаусса распределения значений $\sigma$ для пленочных мононитей СВМПЭ с $\lambda=120$, полученных из гелей в декалине и парафиновом масле, приведенных в работе [29], были построены гистограммы функции плотности вероятности (probability density function - PDF) $\sigma$ при использовании стандартной методики [33]. Полученные результаты приведены на рис. 1 и 2 для мононитей из декалина и парафинового масла, соответственно. Они были аппроксимированы функцией Гаусса при компьютерной обработке. Результаты аппроксимации показаны сплошной огибающей линией.

Как следует из рис. 1 , результат описания гистограммы распределения значений прочности для мононитей на основе декалина при использовании функции Гаусса представляется неудовлетворительным, так как экспериментальные данные не могут быть описаны с помощью единой стандартной колоколообразной кривой. Для мононитей на основе парафинового масла данные также не могут быть корректно описаны подобным образом, хотя результат описания распределения $\sigma$ представляется более корректным по сравнению с рис. 1. Последнее может быть связано с тем, что нити, вытянутые из масла,

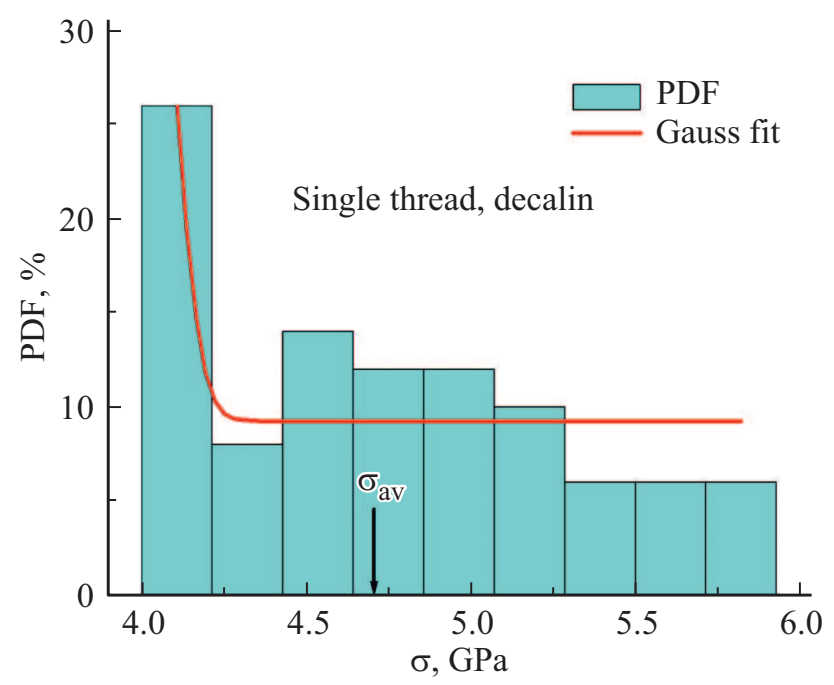

Рис. 1. Функция плотности вероятности PDF в зависимости от прочности при растяжении для сверхпрочных ультраориентированных мононитей СВМПЭ с $\lambda=120$, полученных вытяжкой из гелей в декалине; сплошная линия соответствует аппроксимации функцией Гаусса.

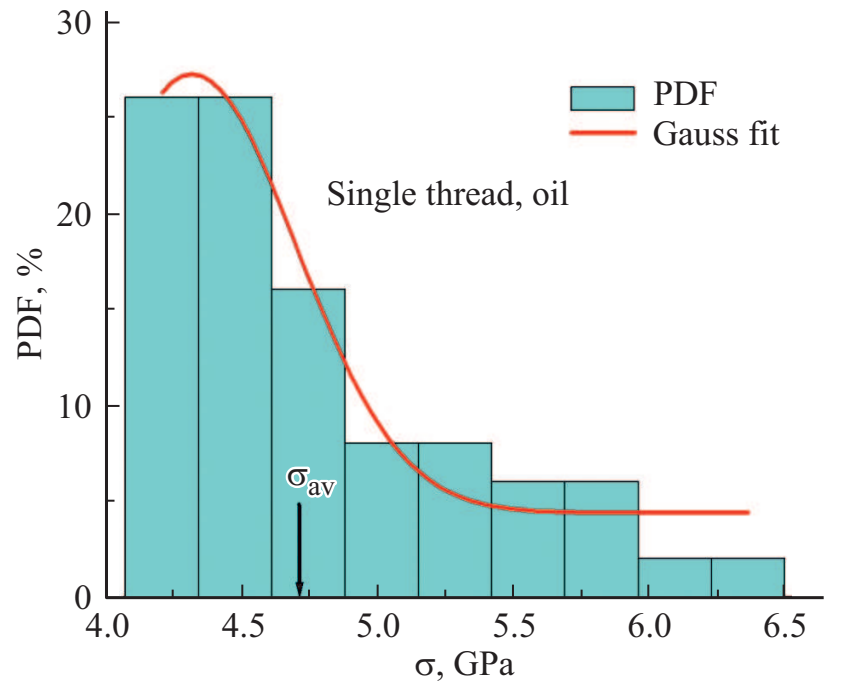

Рис. 2. Функция плотности вероятности PDF в зависимости от прочности при растяжении для сверхпрочных ультраориентированных мононитей СВМПЭ с $\lambda=120$, полученных вытяжкой из гелей в парафиновом масле; сплошная линия соответствует аппроксимации функцией Гаусса.

являются „менее хрупкими“ из-за высокой вероятности присутствия остатков масла в образце, выполняющих роль пластификатора. Тот факт, что распределение прочности для образцов этих двух типов, с одной стороны, не является нормальным, гауссовым, но, с другой стороны, его можно описать в рамках модели Вейбулла [29,30], не представляется удивительным. Это обусловлено тем, что статистика Вейбулла была изначально предложена для анализа процесса разрушения хрупких материалов, а исследуемые нами ультраориентированные нити относятся именно к этому классу материалов.

Проанализируем распределение прочности ориентированных промышленных полифиламентов СВМПЭ. Для анализа в рамках модели Вейбулла измеренные значения $\sigma$ для 50 полифиламентных образцов сначала были сгруппированы в порядке их возрастания (см. рис. 3). Затем эти данные были использованы для построения графика Вейбулла с целью исследования их соответствия уравнениям (3) и (4). Результаты этого анализа представлены на рис. 4. Видно, что зависимость , $\ln \ln \left[1 /\left(1-P_{\mathrm{j}}\right)\right]-\ln \sigma^{\text {“ }}$ представляет собой линейную зависимость, характеризующуюся очень высоким значением коэффициента детерминации $R^{2}=0.99$. Следовательно, проведенный анализ в рамках стандартной функции распределения Вейбулла является корректным. Кроме того, значение $\sigma_{0}=2.39 \mathrm{GPa}$, рассчитанное по уравнению (6) при использовании значения $m=9.9$, определенного из наклона графика на рис. 4, практически совпадает со средним значением $\sigma\left(\sigma_{\text {average }}\right)=2.23 \mathrm{GPa}$, усредненным по результатам всего массива данных 50-ти измерений. Следовательно, проведенный анализ дает результаты, имеющие физический смысл, что являет- 


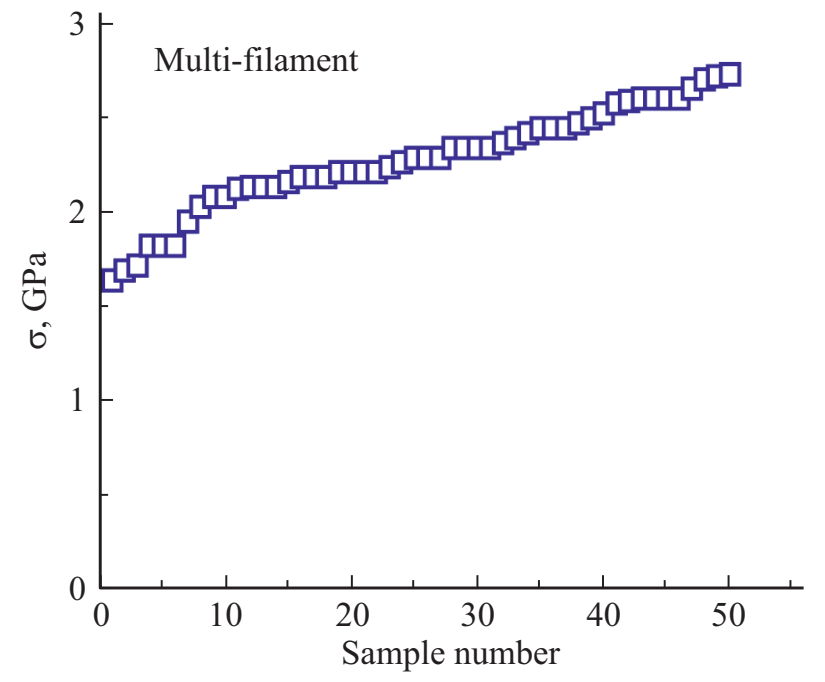

Рис. 3. Прочность при растяжении в порядке возрастания в зависимости от номера образца для промышленных гель-сформованных ориентированных полифиламентов СВМПЭ.

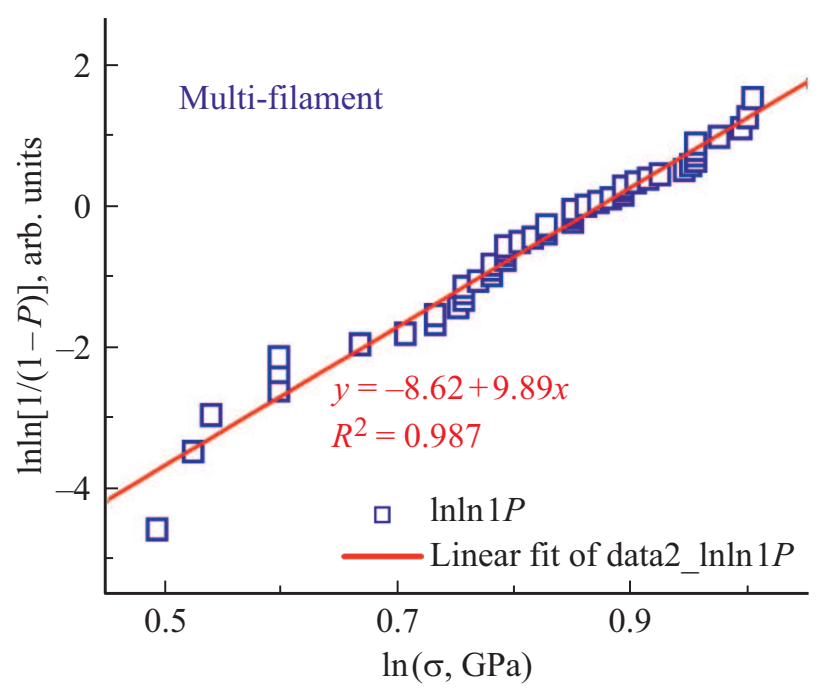

Рис. 4. График Вейбулла для данных рис. 3. Прямой линией показан результат анализа методом наименьших квадратов.

ся дополнительным аргументом в пользу корректности проведенного анализа.

Следует также отметить, что проведенный выше анализ распределения прочности полифиламентов СВМПЭ в рамках модели Вейбулла показал, что данные по прочности для серии из 50 образцов могут быть описаны с помощью стандартной функции распределения Вейбулла с единой прямолинейной зависимостью, характеризующейся наклоном $m=9.9$. Это поведение отличается от поведения мононитей СВМПЭ с $\lambda=120$ [29,30], для которых графики Вейбулла для прочности 50-ти образцов, полученных при использовании различных растворителей, можно было корректно описать лишь при использовании двух зависимостей с наклонами $m=74-76$ для образцов с низкой прочностью $(20 \%$ образцов) и $m=7.2-7.7$ для образцов с высокой прочностью (80\% образцов). Это принципиальное различие при анализе в рамках модели Вейбулла для моно- и полифиламентных образцов свидетельствует о следующем. Как уже отмечалось выше, процесс разрушения полифиламентного образца, состоящего из нескольких сотен отдельных волокон, должен быть статистически более однородным, так как „случайное“ разрушение одного филамента в пучке не должно обязательно приводить к разрушению всего полифиламентного образца, как это происходит в случае мононитей.

Если этот аргумент верен, то описание распределения прочности образцов, состоящих из многочисленных волокон СВМПЭ, в рамках модели Гаусса должно быть более корректным, чем в случае мононитей СВМПЭ с $\lambda=120$ (см. рис. 1 и 2). Действительно, как следует из гистограммы распределения прочности образцов полифиламентов СВМПЭ, приведенной на рис. 5, она может быть представлена в форме единой колоколообразной кривой более корректно по сравнению с мононитями (см. рис. 1 и 2). Кроме того, наибольшая доля измеренных значений прочности $(25 \%)$ располагается в центре кривой, а ее максимум совпадает с $\sigma_{\text {average, что }}$ является характерным для нормального распределения. Следовательно, можно заключить, что, в первом приближении, распределение прочности исследованных высокопрочных полифиламентов СВМПЭ подчиняется также и статистике Гаусса, в отличие от прочности мононитей, для которых модель Гаусса оказалась некорректной.

Что касается значения модуля Вейбулла $m=9.9$, определенного для полифиламента СВМПЭ, то оно сопоставимо с таковыми для пленочных мононитей СВМПЭ с $\lambda=120 \quad(m=7.2-7.7$ для $80 \%$ испытанных в одной серии образцов, имеющих более высо-

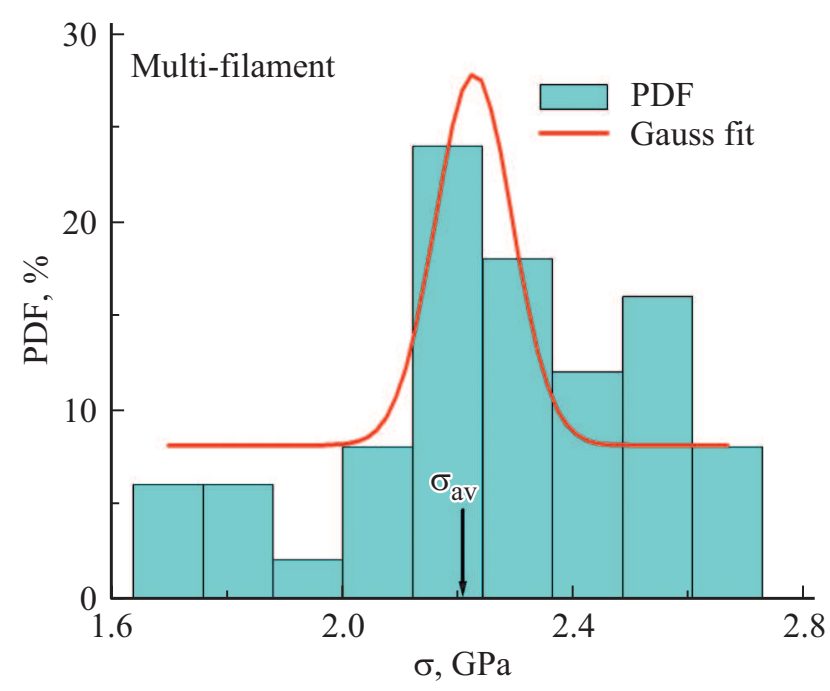

Рис. 5. Функция плотности вероятности PDF в зависимости от прочности при растяжении для данных рис. 3 ; сплошная линия соответствует аппроксимации функцией Гаусса. 
кую прочность), для высокопрочных волокон СВМПЭ Spectra 900 фирмы Honeywell, США $(m=8-12)$ [34], и для таких хрупких материалов неорганического происхождения, как кварцевые $(m=12)$ [9], керамические $(m=8-9)$ [12], стеклянные и углеродные $(m=3-11)[8,15]$ волокна, а также углеродные нанотрубки $(m=3-7)[13]$.

Необходимо отметить, что разброс значений измеренной прочности хрупких материалов как органического, так и неорганического происхождения близок. Однако значение $m=9.9$, определенное в настоящей работе для высокопрочных ориентированных полифиламентных волокон СВМПЭ, существенно, в 4 раза, меньше, чем значение $m=43.4$ для ориентированных полифиламентных волокон полиамида-6 [32], что указывает на заметно меньший разброс данных для последних. Эта разница может быть объяснена тем фактом, что полифиламенты СВМПЭ являются более хрупкими (деформация при разрыве $\varepsilon_{\mathrm{b}} \approx 3 \%$ ) по сравнению с полифиламентами полиамида-6 $\left(\varepsilon_{\mathrm{b}} \approx 15 \%\right)$. Поэтому неудивительно, что для анализа распределения прочности первых более корректной является модель Вейбулла, предложенная для хрупких материалов. В то же время, распределение прочности последних может быть описано как в рамках модели Вейбулла, так и в рамках модели Гаусса. Причиной этого является „дуализм“ деформационного поведения, заключающийся в его „промежуточности“ между хрупким и вязкоупругим, как было предложено в [32].

\section{5. Заключение}

Проведенное в настоящей работе исследование распределения разрывной прочности сверхпрочных ультраориентированных моно- и полифиламентных волокон СВМПЭ, полученных методом гель-формования, при использовании статистических моделей Вейбулла и Гаусса на базе большого массива результатов механических измерений (50 идентичных образцов в каждой из трех серий, т.е. 150 образцов в совокупности) выявило следующие особенности. Показано, что распределение прочности ориентированных полифиламентов СВМПЭ может быть описано как в рамках нормального распределения Гаусса, так и с помощью стандартной функции распределения Вейбулла. В то же время, распределение прочности предельно вытянутых (в 120 крат) мононитей СВМПЭ не удается описать в рамках модели Гаусса. Обнаруженная разница статистического распределения прочности для образцов двух типов связана с критической ролью прорастания в мононити магистральной трещины, инициированной полосой сброса, приводящего к преждевременному разрушению всего образца.

Проведенные нами исследования статистического распределения прочности высокопрочных высокомодульных полимерных материалов различного вида (моно- и полифиламент) и химического строения цепи (карбоцепной СВМПЭ и гетероцепной полиамид-6) показали, что тип статистического распределения, Вейбулла или Гаусса, который окажется наиболее корректным для описания распределения прочности, принципиальным образом зависит от того, является ли образец мононитью или полифиламентом (сотни нитей) [29-32]. Поскольку полагается, что прочность всех высокопрочных высокомодульных материалов в первую очередь контролируется наличием поверхностных трещин (полосы сброса в случае СВМПЭ [29]), распределение Вейбулла, описывающее критический характер хрупкого разрушения, должно быть физически более обоснованным для хрупких моноволокон, как полимерных, так и неорганических.

Кроме того, на основе большого массива статистических данных (измерений также 50 идентичных образцов) для полимерного полифиламентного материала (полиамид-6), состоящего из нескольких сотен отдельных волокон и характеризующегося существенно более низкой прочностью $\sigma \sim 0.8 \mathrm{GPa}$, также был продемонстрирован дуализм статистического распределения прочности [32]. Он заключается в возможности корректного описания одних и тех же экспериментальных результатов в рамках как статистики Гаусса (предполагая, что дефекты распределены равномерно в отдельных нитях по всему объему полифиламентного образца), так и статистики Вейбулла (поскольку деформационное поведение материала оказалось промежуточным между таковыми для хрупких и вязкоупругих материалов). Таким образом, представляется неудивительным, что распределение прочности высокопрочного полифиламентного материала на основе СВМПЭ, исследованного в настоящей работе, с более высокой, но не предельной разрывной прочностью $2.2 \mathrm{GPa}$, все еще удается описать в первом приближении и в рамках модели Гаусса. В то же время, для одиночных нитей из СВМПЭ, характеризующихся заметно более высокой средней прочностью $4.7 \mathrm{GPa}$, модель Гаусса не работает должным образом.

Результаты настоящей работы и наших недавних исследований [29-32] показывают, что, прежде чем провести корректный статистический анализ массива данных механических измерений с использованием определенной статистической модели, следует принимать во внимание характер разрушения исследуемого материала (хрупкий, вязкоупругий или вязкотекучий) и тип образца (моноволокно или полифиламент).

\section{Финансирование работы}

Работа выполнена при финансовой поддержке Российского фонда фундаментальных исследований (гранты 18-29-17023 мк и 19-03-00789).

\section{Конфликт интересов}

Авторы заявляют, что у них нет конфликта интересов. 


\section{Список литературы}

[1] V.A. Marikhin, L.P. Myasnikova. Structural basis of highstrength high-modulus polymers. In: Oriented Polymer Materials / Ed. S. Fakirov. Huthig \& Wepf Verlag-Zug, Heidelberg (1996), p. 38-98.

[2] V. Marikhin, L. Myasnikova, Y. Boiko, E. Ivan'kova, E. Radovanova, P. Yakushev. Role of Reactor Powder Morphology in Producing High-strength High-modulus UHMWPE Fibres. In: Reactor Powder Morphology / Eds L. Myasnikova, P. Lemstra. Nova Publishers, Hauppauge, N.Y., (2011) Ch. 10, p. 235-294.

[3] Yu.M. Boiko, V.V. Kovriga. Int. J. Polym. Mater. 22, 209 (1993).

[4] DSM info, Issued: 20-09-2013, Page: 1/1 Ref.: dtm ya001.

[5] R. Marissen, D. Wienke, R. Homminga, R. Bosman, K.M. Veka, A. Huguet. Mater. Sci. Appl. 7, 238 (2016).

[6] S. Rastogi, Y. Yao, S. Ronca, J. Bos, J. van der Eem. Macromolecules 44, 5558 (2011).

[7] Ю.М. Бойко, В.А. Марихин, О.А. Москалюк, Л.П. Мясникова. ФТТ 61, 182 (2019).

[8] F. Tanaka, T. Okabe, H. Okuda, I.A. Kinloch, R.J. Young. Composites A 57, 88 (2014).

[9] L.G. Baikova, T.I. Pesina, M.F. Kireenko, L.V. Tikhonova, C.R. Kurkjian. Tech. Phys. 60, 869 (2015).

[10] K.-H. Nitta, C.-Y. Li. Physica A 490, 1076 (2018).

[11] W. Weibull. J. Appl. Mech. 18, 293 (1951).

[12] D.M. Wilson. J. Mater. Sci. 32, 2535 (1997).

[13] G. Sun, J.H.L. Pang, J. Zhou, Y. Zhang, Z. Zhan, L. Zheng. Appl. Phys. Lett. 101, 131905 (2012).

[14] J.D. Sullivan, P.H. Lauzon. J. Mater. Sci. Lett. 5, 1245 (1986).

[15] M.R. Gurvich, A.T. Dibenedetto, A. Pegoretti. J. Mater. Sci. 32, 3711 (1997).

[16] N.M. Pugno, R.S. Ruoff. J. Aerospace Eng. 20, 97 (2007).

[17] S. van der Zwaag. J. Test. Eval. 17, 292 (1989).

[18] B. Bergman. J. Mater. Sci. Lett. 3, 689 (1984).

[19] K. Trustrum, A. de S. Jayatilaka. J. Mater. Sci. 14, 1080 (1979).

[20] C.A. Klein. J. Appl. Phys. 101, 124909 (2007).

[21] I.M. de Rosa, J.M. Kenny, D. Puglia, C. Santulli, F. Sarasini. Comp. Sci. Technol. 70, 116 (2010).

[22] Y. Zhang, X. Wang, N. Pan, R. Postle. J. Mater. Sci. 37, 1401 (2002).

[23] H.F. Wu, A.N. Netravali. J. Mater. Sci. 27, 3318 (1992).

[24] Z.P. Bazant, J.-L. Le, M.Z. Bazant. Proc. Acad. Sci. USA 106, 11484 (2009).

[25] Z.P. Bazant, S.-D. Pang. Proc. Acad. Sci. USA 103, 9434 (2006).

[26] A.H. Barber, R. Andrews, L.S. Shaudler, H.D. Wagner. Appl. Phys. Lett. 87, 203106 (2005).

[27] A. Roy, S. Chakraborty, S.P. Kundu, R.K. Basak, S.B. Majumber, B. Adhikari. Biores. Technol. 107, 222 (2012).

[28] A. de S. Jayatilaka, K. Trustrum. J. Mater. Sci. 12, 1426 (1977).

[29] Yu.M. Boiko, V.A. Marikhin, L.P. Myasnikova, O.A. Moskalyuk, E.I. Radovanova. J. Mater. Sci. 52, 1727 (2017).

[30] Ю.М. Бойко, В.А. Марихин, О.А. Москалюк, Л.П. Мясникова, Е.И. Радованова. ФТТ 58, 2065 (2016).
[31] Yu.M. Boiko, V.A. Marikhin, L.P. Myasnikova, E.I. Radovanova. Colloid Polym. Sci. 296, 1651 (2018).

[32] Ю.М. Бойко, В.А. Марихин, О.А. Москалюк, Л.П. Мясникова, Е.С. Цобкалло. Письма ЖТФ 45, 8, 37 (2019).

[33] Е.И. Куликов. Прикладной статистический анализ. Радио и связь, М. (2008), 464 с.

[34] P. Schwartz, A. Netravali, S. Sembach. Textile Res. J. 56, 502 (1986).

Редактор Т.Н. Василевская 